\title{
Cannabinoids for Symptom Management and Cancer Therapy: The Evidence
}

\author{
Mellar P. Davis, MD
}

\begin{abstract}
Cannabinoids bind not only to classical receptors (CB1 and CB2) but also to certain orphan receptors (GPR55 and GPR119), ion channels (transient receptor potential vanilloid), and peroxisome proliferator-activated receptors. Cannabinoids are known to modulate a multitude of monoamine receptors. Structurally, there are 3 groups of cannabinoids. Multiple studies, most of which are of moderate to low quality, demonstrate that tetrahydrocannabinol (THC) and oromucosal cannabinoid combinations of THC and cannabidiol (CBD) modestly reduce cancer pain. Dronabinol and nabilone are better antiemetics for chemotherapy-induced nausea and vomiting (CINV) than certain neuroleptics, but are not better than serotonin receptor antagonists in reducing delayed emesis, and cannabinoids have largely been superseded by neurokinin-1 receptor antagonists and olanzapine; both cannabinoids have been recommended for breakthrough nausea and vomiting among other antiemetics. Dronabinol is ineffective in ameliorating cancer anorexia but does improve associated cancer-related dysgeusia. Multiple cancers express cannabinoid receptors directly related to the degree of anaplasia and grade of tumor. Preclinical in vitro and in vivo studies suggest that cannabinoids may have anticancer activity. Paradoxically, cannabinoid receptor antagonists also have antitumor activity. There are few randomized smoked or vaporized cannabis trials in cancer on which to judge the benefits of these forms of cannabinoids on symptoms and the clinical course of cancer. Smoked cannabis has been found to contain Aspergillosis. Immunosuppressed patients should be advised of the risks of using "medical marijuana" in this regard.
\end{abstract}

J Natl Compr Canc Netw 2016;14(7)915-922

\section{Introduction}

Cannabinoids have been used throughout history for many ailments. Naturally occurring cannabis sativa contains multiple cannabinoids that have medicinal benefits. The medicinal benefits are either related to a single cannabinoid or caused by interactions between cannabinoids (called an entourage effect). Tetrahydrocannabinol (THC) is the major constituent of cannabis. Besides cannabinoids, cannabis sativa also contains terpenes and phenolic compounds that may have medicinal benefits. Marijuana, the dried leaves and flowers of the hemp plant that are smoked as a drug, was on the American pharmacopeia until 1944. It returned in the form of delta(9)THC (dronabinol) in 1986, approved for treatment of chemotherapy-induced nausea and vomiting. ${ }^{1}$

From Cleveland Clinic Lerner School of Medicine, Case Western University, and Palliative Medicine and Supportive Oncology Services, Division of Solid Tumor, Taussig Cancer Institute, The Cleveland Clinic, Cleveland, Ohio. Submitted November 12, 2015; accepted for publication May 4, 2016.

\section{Classical and Nonclassical Cannabinoids and Receptors}

Cannabinoids bind to classical receptors (CB1 and CB2) as agonists, antagonists, or inverse agonist (Figure 1). Cannabinoids also bind to certain orphan receptors (GPR55 and GPR119), ion channels (transient receptor potential vanilloid [TPRV]), and peroxisome proliferator-activated receptors (PPARs), and modulate a multitude of monoamine receptors through heterodimer formation between monoamine and cannabinoid receptors. ${ }^{2,3}$ The biologic activity of a single cannabinoid depends on interactions with a multitude of cannabinoid and noncannabinoid receptors and ion channels. Cannabinoids also have an entourage effect in that a combination of 2 or more cannabinoids produce a syn-
The author has disclosed that he has no financial interests, arrangements, affiliations, or commercial interests with the manufacturers of any products discussed in this article or their competitors.

Correspondence: Mellar P. Davis, MD, 14 Red Oak Drive, Danville, PA 17821-8417. E-mail: Banpangur70@gmail.com 

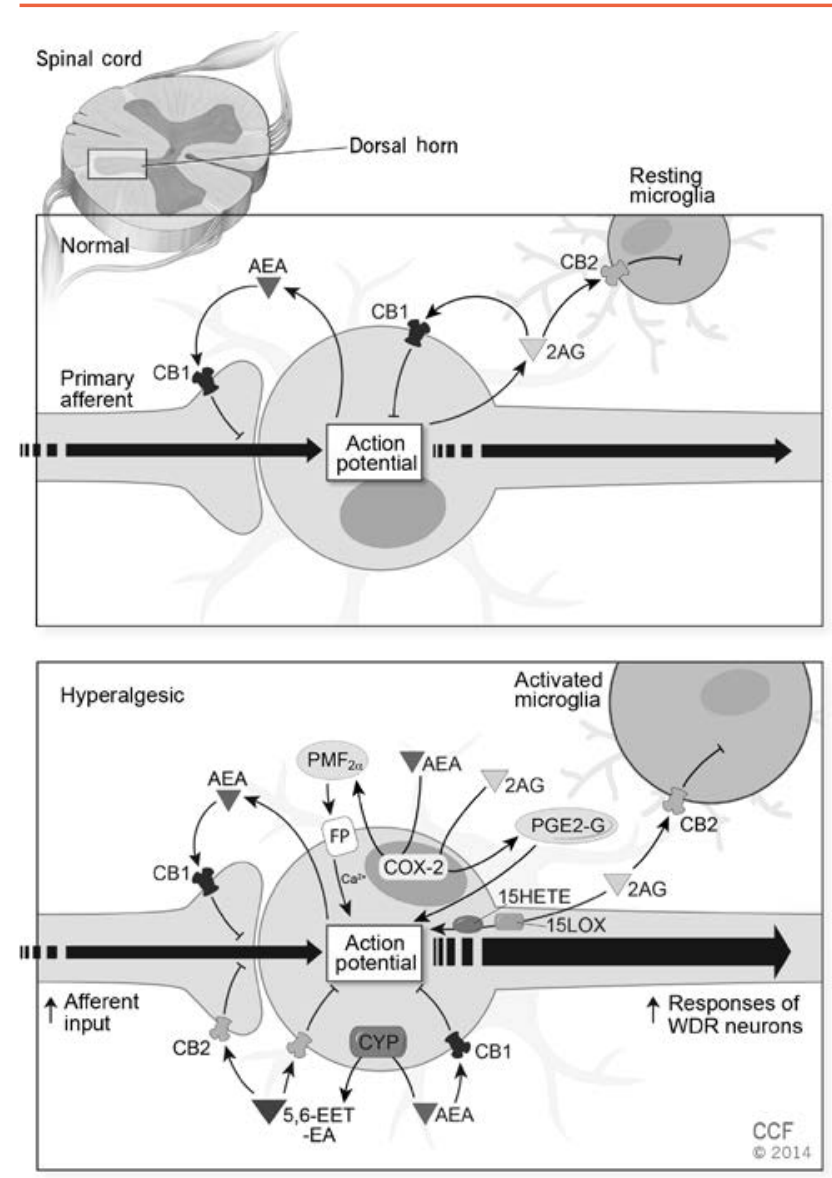

Figure 1 Endocannabinoids are produced from arachidonic acid upon activation of neurons. Postsynoptic production of 2 main endocannabinoids, anandamide (AEA), and 2-archidonoylglycerol (2AG) is followed by extracellular release and binding to 2 major cannabinoid receptors (CB1 and CB2). CB1 dampens neuronal depolarization and release of neurotransmitter, whereas CB2 bands to immunocytes (glia), dampening neuroinflammation. AEA and 2AG are produced and metabolized by several different routes. Classically, AEA is catabolized by fatty acid amide hydrolase- 1 and monoacylglycerol lipase for 2AG. However, both endocannabinoids can be metabolized by cyclooxygenase-2 (COX-2), lipoxygenase (15LOX), and cytochromes P450 (CYP). Cyclooxygenases produce pronociceptive prostamides (PMF2 $\alpha$ ) and prostaglandins(PGE2-G), which can bind to prostamide F2a receptors (FP) causing

hyperalgesia, whereas lipooxygenase also produces a pronociceptive metabolite (15HETE). Cytochromes transform AEA into a potent and selective CB2 agonist (5, 6-EET-EA).

Abbreviation: WDR, wide dynamic range.

ergistic biologic effect either through improved benefits or reduced toxicity. ${ }^{4}$

Structurally, there are 3 groups of cannabinoids. The first group is endogenous; largely anandamide (AEA) and 2-arachidonoylglycerol (2AG). These are derived from membrane arachidonic acid and are mainly agonists to CB1 and CB2 receptors. The second group are phytocannabinoids represented by THC and cannabidiol (CBD), with other minor but important phytocannabinoids in marijuana and commercial hemp. The third group comprises syn- thetic cannabinoids represented by dronabinol and nabilone. $^{5}$

Certain phytocannabinoids, such as CBD and the endogenous cannabinoid palmitoylethanolamine (which is also available as a nutraceutical in certain countries), have no psychotomimetic effects, which makes them attractive as therapeutic drugs. Cannabidiol, isolated in 1963, is a negative allosteric inhibitor of CB1. In animal models, CBD has antiemetic, analgesic, anxiolytic, anti-inflammatory, and antipsychotic effects. ${ }^{6}$ Cannabidiol inhibits cyclooxygenase and lipoxygenases and reduces $\mathrm{N}$-methyl-D-aspartate toxicity. It also activates TRPV1.? Palmitoylethanolamine (PEA) was first described in 1957 and has been used to treat neuropathic pain (largely entrapment neuropathies) and other chronic pain phenotypes. PEA inhibits cyclooxygenase, inducible and endothelial nitric oxide synthase, and GPR55 and GPR119, and interacts with TRPV1 and PPAR- $\alpha$. PEA reduces neuroinflammation and overactivation of astrocytes and glial cells, which is likely the mechanism behind PEA benefits in relieving neuropathic pain. ${ }^{8,9}$ There is a large clinical experience with PEA (30 clinical trials, 6,000 patients), although none centered on cancer pain. The 8 trials that demonstrated significant reductions in nerve compression pain used doses ranging from 300 to $1,200 \mathrm{mg} / \mathrm{d}$. Adverse effects are rarely seen with PEA. ${ }^{10,11}$

Classical cannabinoid receptors are G-proteincoupled receptors, which, when activated, inhibit adenylyl cyclase and calcium channels and activate rectifying potassium channels and mitogen active protein kinase. ${ }^{3} \mathrm{CB} 1$ receptors are found on presynaptic afferent neurons; activated receptors dampen excessive neurotransmission as a retrograde synaptic negative modulator. Endogenous cannabinoids are not stored in vesicles but rather are synthesized from membrane arachidonic acid on neuron activation of postsynaptic neurons. This generates AEA and 2AG, which bind to presynaptic receptors. ${ }^{12} \mathrm{CB} 2$ receptors are largely found on immunocytes, including glia, and modulate cytokine expression and cell migration. CB2 receptors are sparsely expressed on neurons relative to CB1 receptors. ${ }^{13,14}$

Activation of CB1 receptors is responsible for the psychotomimetic adverse effects of cannabis. Cannabidiol as an inverse agonist to $\mathrm{CB} 1$ receptors blocks the psychotomimetic adverse effects of THC. ${ }^{15}$ Highly selective CB2 receptor agonists do not produce psychotomimetic effects but can be immunosuppressive. ${ }^{16}$ 


\section{Pharmacokinetics of Cannabinoids}

The bioavailability of smoked or vaporized THC in marijuana is $10 \%$ to $25 \%$, with a half-life distribution phase of 0.5 hours. Bioavailability depends on breath hold, duration of breath hold, and depth of inhalation. ${ }^{17}$ Peak serum concentrations occur within 2 to 10 minutes. Oral bioavailability of THC and CBD ranges between 6\% and 20\%. Delta(9)THC is metabolized to an active metabolite (11-hydroxy-THC). Because delta(9)-THC is lipophilic, it has a large volume of distribution. The half-life is 3 to 4 hours, but the terminal half-life is 25 to 36 hours. ${ }^{17}$ Delta(9)-THC is metabolized by 2 cytochromes, CYP3A4 and CYP2D6, and subsequently glucuronidated. ${ }^{18,19}$ Cannabidiol in vitro inactivates CYP3A4; however, nabiximols mouth spray, which contains THC and CBD $(2.7 \mathrm{mg}$ of THC and $2.5 \mathrm{mg}$ of CBD), does not adversely influence THC clearance. Certain cannabinoids induce $\mathrm{P} 450$ isoforms CYP1A2, CYP2C, and CYP3A. However, cannabis tea has not been found to clinically alter irinotecan or docetaxel pharmacokinetics. ${ }^{20}$

\section{Cannabinoids in Practice}

In a study that permitted patients to register for the use of cannabis to relieve cancer symptoms, the most common reasons given were pain, well-being, appetite, and nausea. ${ }^{21}$ Most of these patients (84\%) had metastatic cancer and died within 6 months of receiving the permit. All but 10\% smoked cannabis as the method of delivery. Only 42\% listed their oncologist as providing information about cannabis for their symptoms. In a survey of Québec physicians, 22 27\% prescribed cannabis for various reasons, and $23 \%$ for chronic noncancer pain. Most who prescribed cannabis did so for 5 or fewer patients. The principal reason for a cannabinoid prescription was physician comfort (odds ratio [OR], 1.25). The authors concluded that guidelines and education regarding cannabis use were needed.

\section{Cannabinoids in the Management of Symptoms}

A large systematic review of cannabis use for symptoms was published in 2015. Benefits and effect size was assessed in multiple medical illnesses. The review involved 79 randomized trials and 6,462 pa- tients. Individuals with cancer made up a minority of participants. Dronabinol and nabilone improved chemotherapy-related nausea and vomiting relative to older neuroleptics in studies reported in the 1970 s and 1980 s (42\% vs 20\%; OR, 3.8, 95\% CI, $1.55-9.42 ; 3$ studies). Cannabinoids reduced pain in general (37\% vs 31\%; OR, 1.4; 95\% CI, 0.99-2.0; 8 trials). In these studies, cannabinoids reduced pain intensity by 0.46 points on a 0 to 10 numerical rating scale (NRS) (95\% CI, -0.80 to $-0.11 ; 5$ trials). However, in the review, adverse events were 3 times greater with cannabinoids than with placebo (OR, 3.03; 95\% CI, 2.4-3.80). The strength of the evidence by GRADE (grades of recommendation, assessment, development, and evidence) standards was moderate for pain and weak for the remainder of the symptoms. ${ }^{23}$

\section{Cannabinoids and Cancer Pain}

Two studies published in 1975 compared dronabinol with placebo for cancer pain. ${ }^{24,25}$ A $10-\mathrm{mg} / \mathrm{d}$ dosage of dronabinol was better tolerated than $20 \mathrm{mg} / \mathrm{d}$. Side effects included dizziness, somnolence, ataxia, and blurred vision. Dronabinol at 15 and $20 \mathrm{mg} / \mathrm{d}$ provided superior pain relief over placebo, and provided equivalent analgesia as 60 and $120 \mathrm{mg}$ of oral codeine, respectively.

A recent systematic review of cannabinoids for pain included 18 placebo-controlled trials, of which 4 involved patients with cancer. The standard mean difference in pain was -0.61 on a 0 to 10 NRS ( $95 \%$ $\mathrm{CI},-0.37$ to -0.84$)$. The authors noted bias in trials that favored the treatment arm. Cannabinoids were much more likely to result in adverse effects (OR, 4.5; 95\% CI, 3.0-6.6), including altered perception and impaired psychomotor and cognitive function. ${ }^{26}$

Two recent trials have used nabiximols, which is an oromucosal spray containing $2.7 \mathrm{mg}$ of THC and $2.5 \mathrm{mg}$ of CBD per $100 \mathrm{mcL}$. Patients had cancer pain poorly controlled with opioids. In the first study, individuals with cancer were on daily morphineequivalent doses of $271 \mathrm{mg}$. There were 2 outcomes in the first study: reduction in pain severity and reduction in breakthrough rescue doses. The average dose of nabiximols was 8.75 sprays per day $(24 \mathrm{mg}$ THC). The difference in pain severity by numerical rating scale was $-0.67(P=.014)$, and adjusted differences between groups were $-0.055(P=.024)$. The 
$30 \%$ response rate for nabiximols, placebo, and the number needed to treat (NNT; the number of individuals needed to treat to reduce pain severity in one individual by $30 \%$ ) were $43 \%$ with nabiximols and $21 \%$ with placebo, and 4.7 , respectively. Interestingly, there was no pain relief at THC doses greater than a $22.5 \mathrm{mg} / \mathrm{d} .{ }^{27}$

The second trial published in 2012 involved patients assigned to 4 treatment groups: placebo, low dose (1-4 sprays per day), medium dose (6-10 sprays per day), and high-dose (11-16 sprays per day) nabiximols. This was a short study of 5 weeks. The median morphine daily equivalent for the group was $120 \mathrm{mg}$. This study failed to meet the primary outcome of $30 \%$ reduction in pain severity. Some benefit was noted in reduction of pain severity in the lower- and mediumdose groups but not in the high-dose group $(-0.75$ with the low dose; -0.36 with the medium dose). ${ }^{28}$ Both studies demonstrated a dose ceiling effect with pain. The second study did not demonstrate benefits to breakthrough pain. There was some improvement in sleep, but also an increase in nausea with nabiximols, particularly at the high dose.

Neuropathic pain is common in cancer. Nabiximols was investigated in an extension of 2 randomized trials. ${ }^{29} \mathrm{Up}$ to 24 doses per day could be used and the duration of study was 38 weeks. The primary outcome was reduction in pain intensity according to an NRS, with secondary outcomes being response defined as a $30 \%$ or $50 \%$ reduction in pain intensity. The neuropathic pain scale, sleep quality, and quality of life (using the EQ-5D questionnaire) were also assessed, as was drug toxicity. Individuals were kept on their prestudy analgesics.

A total of 308 patients were screened and 234 patients completed the study. Patients who had previously been on a placebo in the randomized trial had a discontinuation rate of $27 \%$, whereas those who had received active drug during the randomized trial had an $11 \%$ discontinuation rate; discontinuation was largely for adverse effects. Pain intensity by NRS decreased from $5.5(0-10)$ to 4.2 over the 9 -month period. A total of $28 \%$ were responders ( $\geq 30 \%$ reduction in pain intensity). Approximately $75 \%$ reported some improvement in pain intensity, $22 \%$ had no change, and $8 \%$ worsened. Doses on average were taken 6 to 8 per day. Most adverse effects occurred in less than $10 \%$ of patients and only $1 \%$ had serious adverse events related to the drug.
Maximum response occurred between weeks 14 and 26 , and by week 38 , pain began to increase. Sleep quality and quality of life, which had improved on the randomized trial, were maintained in the openlabel study. ${ }^{29}$

\section{Cannabis in Chemotherapy-Induced Nausea and Vomiting}

A systematic review of antiemetics in older trials found that oral dronabinol, nabilone, and intramuscular levonantradol were better antiemetics than certain neuroleptics. ${ }^{30}$ In 30 randomized trials in this review, the NNT to benefit one patient with nausea was 6 and the NNT for vomiting was 8. A second systematic review of published trials included 15 studies. ${ }^{31}$ Nabilone was a better antiemetic than prochlorperazine, domperidone, and alizapride. Cannabinoids were as effective as oral metoclopramide and haloperidol. There were no comparisons between cannabinoids and serotonin receptor antagonists (5HT3 antagonists). Mechanistically, cannabinoids cause negative allosteric modulation of 5HT3 receptors, and therefore may be 5HT3 receptor antagonists. ${ }^{32}$ This may account for why combinations of dronabinol and ondansetron are not better than either agent alone. ${ }^{33}$ Nabiximols has been added to corticosteroids plus a 5HT3 antagonist or metoclopramide for moderate emetogenic chemotherapy in a small randomized trial of 16 patients, 9 of whom receive placebo. ${ }^{34}$ Five of 7 patients receiving the nabiximols had a complete or partial response, whereas 2 of 9 patients treated with placebo experienced a response. This was a small underpowered study and did not include neurokinin 1 receptor antagonists.

There are 3 small trials involving smoked cannabis in the treatment of chemotherapy-induced nausea and vomiting. ${ }^{31}$ Two studies switched to smoked cannabis for patients with no response to dronabinol, and one study was a crossover comparison between dronabinol and smoked cannabis. Overall, 25\% had a positive response, $35 \%$ preferred dronabinol, $20 \%$ preferred smoked cannabis, and $45 \%$ had no preference. ${ }^{31}$ Average doses in reported trials are nabilone at 1 to $2 \mathrm{mg}$ twice daily and dronabinol at $5 \mathrm{mg}$ every 2 to 4 hours, up to 4 to 6 doses a day. ${ }^{35,36}$

Recent studies have found olanzapine to be superior to metoclopramide in breakthrough delayed 
nausea and vomiting from chemotherapy. ${ }^{37,38}$ Olanzapine is as effective as aprepitant in reducing delayed emesis, and superior in reducing delayed nausea. Indirect comparisons are fraught with hazards; however, the newer antiemetics appear to be superior to cannabinoids, although not formally compared in a randomized trial. ${ }^{37,38}$ None of the guidelines developed by the NCCN, Multinational Association of Supportive Care in Cancer (MASCC), ASCO, and ESMO recommend cannabinoids as first-line antiemetics for chemotherapy-induced nausea and vomiting, nor have cooperative groups conducting antiemetic trials pursued further cannabinoid trials. ${ }^{39,40}$

A systematic review of cannabinoids for patients experiencing refractory nausea and vomiting from chemotherapy was published recently. ${ }^{41}$ The search involved the electronic database from inception to January 2015. Twenty-three trials were reviewed; most were crossover studies. Chemotherapy regimens were moderate to highly emetogenic. Trials were between 1975 and 1991. No comparison has been performed between cannabinoids and newer antiemetics such as the 5HT3 receptor antagonists. Cannabinoids were superior to placebo for complete absence of vomiting (relative risk [RR], 5.7; 95\% CI, 2.6-12.6; low-quality evidence). There were no differences between prochlorperazine and cannabinoids for nausea (RR, 1.5; 95\% CI, 0.67-3.2; lowquality evidence) and vomiting (RR, $1.11 ; 95 \% \mathrm{CI}$, 0.86-1.44). Rates of withdrawal from study for adverse effects and lack of efficacy were greater with cannabinoids. Comparisons between cannabinoids and other antiemetics, such as metoclopramide, domperidone, and chlorpromazine, were insufficient to make any conclusions. Cannabinoids may be useful for treating refractory chemotherapy-induced nausea and vomiting, but are unlikely to be better than standard less-expensive antiemetics.

The evidence for cannabinoids in advanced cancer is even less compelling. Individuals with advanced cancer and nausea should be treated with metoclopramide or haloperidol as first-line therapy. Second-line therapy should be either a 5HT3 receptor antagonist or olanzapine. There are no prospective studies of smoked or vaporized cannabis, partly because of a lack of government funding, to show benefits rather than harm. ${ }^{42}$

\section{Cannabinoids in Cancer-Associated Anorexia}

Two small early trials demonstrated that THC improved appetite in patients with cancer and slowed weight loss. ${ }^{31}$ There was additional evidence from a small case series. ${ }^{43}$ In a large 3-arm randomized trial, comparison was made between oral megestrol acetate, $800 \mathrm{mg} / \mathrm{d}$; dronabinol, $2.5 \mathrm{mg}$ twice daily; and the combination of megestrol plus dronabinol. Oral megestrol was superior to dronabinol (75\% vs $49 \%$, respectively) when appetite was assessed by the Functional Assessment of Anorexia/Cachexia Therapy questionnaire. The addition of dronabinol did not improve megestrol responses. ${ }^{44}$

A second study performed by the Cannabis-InCachexia-Study-Group compared dronabinol with or without CBD and placebo in patients with advanced cancer. The 3 arms were dronabinol, $2.5 \mathrm{mg}$ twice daily; dronabinol, $2.5 \mathrm{mg}$ twice daily plus CBD, $1 \mathrm{mg}$ twice daily; and placebo. The trial duration was 6 weeks. Outcomes were appetite, mood, nausea, and quality of life by the EORTC quality of life questionnaire-C30. Of 243 randomized individuals, 164 completed the study. Almost one-third (32\%) had lost at least $10 \%$ of their body weight prior to entering the study; the average appetite was 31 on a $100-\mathrm{mm}$ visual analog scale at baseline. Intention-to-treat analysis demonstrated no difference in appetite, nausea, weight, or quality of life between arms of the study. An independent review board closed the study for reasons of futility. ${ }^{45}$ The issue with the 2 randomized trials is that the dose of dronabinol may have been too low to see a benefit.

In recent developments, olanzapine has been added to megestrol and compared with megestrol alone in a randomized trial. The combination significantly improved appetite, nausea, and weight compared with megestrol alone. ${ }^{46}$ By indirect comparison, it appears that the combination of olanzapine and megestrol is superior to megestrol in managing cancer-related anorexia and weight loss, whereas dronabinol does not add to the benefits of megestrol. Recent randomized trials of cancer-related anorexia and cachexia have not included cannabinoids in treatment arms. ${ }^{47}$ There are no randomized trials of smoked cannabis in the management of cancer anorexia. 


\section{Cannabinoids and Dysgeusia}

Dysgeusia is a common complaint during chemotherapy and in those with advanced cancer. Qualitative taste changes lead to nutritional compromise. Zinc supplements ( $50 \mathrm{mg}, 3$ times daily) has the best evidence for benefit. ${ }^{48} \mathrm{~A}$ small randomized trial compared dronabinol at $2.5 \mathrm{mg}$ twice daily with placebo in individuals with dysgeusia. ${ }^{49}$ The duration of the trial was only 18 days. A taste and smell survey, a 3-day food record, appetite and macronutrient preferences were assessed as outcomes. Quality of life was a secondary outcome. Dronabinol improved and enhanced chemosensory perception $(P=.026$ and $P<.001$, respectively), improved the taste of food $(P=.004)$, increased pre-meal appetite, and increased proportion of calories consumed as protein $(P=.05$ and $P=.008$, respectively) compared with placebo. Secondary outcomes included improved sleep $(P=.025)$ and quality of life. ${ }^{49}$

\section{Cannabinoids as Treatment of Advanced Cancer}

There is a large body of in vitro and in vivo preclinical cancer model evidence that cannabinoids block cancer proliferation signaling, tumor migration and cell division, and angiogenesis. ${ }^{50,51}$ In general, cancers express cannabinoid receptors to a greater extent than do native tissues. ${ }^{52}$ Expression of cannabinoid receptors directly correlates with tumor grade. ${ }^{53}$ The cannabinoid-induced apoptotic and antiproliferative effects demonstrated in animal models are both cannabinoid receptor-dependent and cannabinoid receptor-independent; benefits may depend on an entourage effect between various cannabinoids. ${ }^{54}$ Unfortunately, there is only a single small phase I clinical trial that investigated local injections of THC in patients with gliomas. ${ }^{55}$

On the other hand, clinicians should be cautious in recommending cannabis to patients with cancer, because expression of cannabinoid receptors on tumors can be a survival mechanism. ${ }^{56}$ Paradoxically, blocking CB1 receptors inhibits a multitude of cancers. This observation and the consistent finding of a high expression of $\mathrm{CB}$ receptors on cancers supports an alternative but not mutually exclusive view of $\mathrm{CB}$ receptors as promoters of cancer, and $\mathrm{CB} 1$ receptor antagonists as having antitumor effects. ${ }^{56-61}$ Submicromolar cannabinoid concentrations can stimulate cancer cell lines. Cannabinoids that interact with CB2 receptors are immunomodulatory and immunosuppressive. THC has been shown to stimulate tumor growth in immunocompetent animals. ${ }^{62}$ This may be a particularly important issue in patients receiving checkpoint inhibitor therapy.

Exogenous cannabinoids can be immunostimulating or immunosuppressive. Low doses of THC stimulate $\mathrm{T}$ cells; high doses of THC are immunosuppressive in humans. THC modulates the T helper 1 and T helper 2 (Th1/Th2) balance to favor Th2 responses. ${ }^{63}$ Th2 CD8 tumor-infiltrating lymphocytes (TILs) have been noted to be impaired in human tumors. ${ }^{64}$ Programmed death-ligand 1 (PD-L1) expression in tumor cells could be the result of an antitumor immune response that includes production of interferon gamma and other inflammatory factors, which in turn upregulates PD-L1. ${ }^{65}$ PD-L1 in turn prevents innate and adaptive cellular distraction of tumor. PD-L1 is found on both CD4 and CD8 TILs and predicts responses to anti-PD-L1 antibodies. ${ }^{66}$ Currently, it is not known whether THC alterations in Th1/Th2 immunity will either improve or impair responses to checkpoint inhibitors and whether these effects are dose-related.

Smoked cannabis contains Aspergillus; invasive aspergillosis has been described in marijuana smokers undergoing renal transplant, treated for leukemia or solid tumors, with AIDs, and undergoing chronic steroid therapy ${ }^{67}$ For this reason, the use of smoked cannabis in individuals receiving chemotherapy should be discouraged. Patients should be informed about the risk of cannabinoid use.

\section{Conclusions}

Cannabinoids have been found to marginally improve cancer pain. There appears to be a THC ceiling dose at 20 to $25 \mathrm{mg}$. The antiemetic activity of cannabinoids is matched by $5 \mathrm{HT} 3$ antagonists (and is likely through 5HT3 receptors). Serotonin receptor antagonists have a better therapeutic index due to fewer side effects. Cannabinoid antiemesis is surpassed by olanzapine, which is now included in NCCN Guidelines. Although cannabinoids are effective in improving appetite in individuals with AIDs, in randomized trials, dronabinol is inferior to megestrol and is no better than placebo in patients with cancer. The combination of olanzapine plus 
Cannabinoids for Symptom Management and Cancer Therapy: The Evidence

megestrol is far better therapy than cannabinoids. Dronabinol has been shown to improve dysgeusia, but the evidence is from one small trial. Zinc remains the first line of therapy for dysgeusia. Additional trials of dronabinol for dysgeusia should be performed to confirm the response. A significant body of evidence shows that cannabinoids may be effective in the treatment of cancer, but no randomized trials have been performed. There is also evidence that cancers may depend on cannabinoid receptors for survival and that cannabinoids stimulate tumor proliferation and migration.

\section{References}

1. Ungerleider JT, Sarna G, Fairbanks LA, et al. THC or compazine for the cancer chemotherapy patient-the UCLA study. Part II: patient drug preference. Am J Clin Oncol 1985;8:142-147.

2. Ryberg E, Larsson N, Sjogren S, et al. The orphan receptor GPR55 is a novel cannabinoid receptor. Br J Pharmacol 2007;152:1092-1101.

3. Demuth DG, Molleman A. Cannabinoid signalling. Life Sci 2006;78:549_ 563.

4. Ben-Shabat S, Fride E, Sheskin T, et al. An entourage effect: inactive endogenous fatty acid glycerol esters enhance 2-arachidonoyl-glycerol cannabinoid activity. Eur J Pharmacol 1998;353:23-31.

5. Grotenhermen F. Cannabinoids. Curr Drug Targets CNS Neurol Disord 2005;4:507-530.

6. Mechoulam R, Parker LA, Gallily R. Cannabidiol: an overview of some pharmacological aspects. J Clin Pharmacol 2002;42(11 Suppl):11S-9S.

7. Zuardi AW, Crippa JA, Hallak JE, et al. A critical review of the antipsychotic effects of cannabidiol: 30 years of a translational investigation. Curr Pharm Des 2012;18:5131-5140.

8. Skaper SD, Facci L. Mast cell-glia axis in neuroinflammation and therapeutic potential of the anandamide congener palmitoylethanolamide. Philos Trans R Soc Lond B Biol Sci 2012;367:3312-3325.

9. Mattace Raso G, Russo R, et al. Palmitoylethanolamide in CNS health and disease. Pharmacol Res 2014;86:32-41.

10. Bettoni I, Comelli F, Colombo A, et al. Non-neuronal cell modulation relieves neuropathic pain: efficacy of the endogenous lipid palmitoylethanolamide. CNS Neurol Disord Drug Targets 2013;12:34-44.

11. Gatti A, Lazzari M, Gianfelice V, et al. Palmitoylethanolamide in the treatment of chronic pain caused by different etiopathogenesis. Pain Med 2012;13:1121-1130

12. Pertwee RG. Ligands that target cannabinoid receptors in the brain: from THC to anandamide and beyond. Addict Biol 2008;13:147-159.

13. Griffin G, Fernando SR, Ross RA, et al. Evidence for the presence of CB2like cannabinoid receptors on peripheral nerve terminals. Eur J Pharmacol 1997;339:53-61.

14. Pertwee RG. Pharmacology of cannabinoid $C B 1$ and $C B 2$ receptors. Pharmacol Ther 1997;74:129-180.

15. Zuardi AW, Hallak JE, Crippa JA. Interaction between cannabidiol (CBD) and (9)-tetrahydrocannabinol (THC): influence of administration interval and dose ratio between the cannabinoids. Psychopharmacology (Berl) 2012;219:247-249.

16. Basu S, Dittel BN. Unraveling the complexities of cannabinoid receptor 2 (CB2) immune regulation in health and disease. Immunol Res 2011;51:26-38.

17. Grotenhermen F. Pharmacokinetics and pharmacodynamics of cannabinoids. Clin Pharmacokinet 2003;42:327-360.

18. Pertwee RG. The pharmacology of cannabinoid receptors and their ligands: an overview. Int J Obes (Lond) 2006;30(Suppl 1):S13-18.

19. Pertwee RG. Pharmacology of cannabinoid receptor ligands. Curr Med Chem 1999;6:635-664.

20. Engels FK, de Jong FA, Sparreboom A, et al. Medicinal cannabis does not influence the clinical pharmacokinetics of irinotecan and docetaxel. Oncologist 2007;12:291-300.
21. Waissengrin B, Urban D, Leshem $Y$, et al. Patterns of use of medical cannabis among Israeli cancer patients: a single institution experience. J Pain Symptom Manage 2015;49:223-230.

22. St-Amant $H$, Ware MA, Julien N, Lacasse A. Prevalence and determinants of cannabinoid prescription for the management of chronic noncancer pain: a postal survey of physicians in the Abitibi-Temiscamingue region of Quebec. CMAJ Open 2015;3:E251-257.

23. Whiting PF, Wolff RF, Deshpande S, et al. Cannabinoids for medical use: a systematic review and meta-analysis. JAMA 2015;313:2456-2473.

24. Noyes R Jr, Brunk SF, Baram DA, Canter A. Analgesic effect of delta-9. tetrahydrocannabinol. J Clin Pharmacol 1975;15:139-143.

25. Noyes R Jr, Brunk SF, Avery DA, Canter AC. The analgesic properties of delta-9-tetrahydrocannabinol and codeine. Clin Pharmacol Ther 1975;18:84-89.

26. Martin-Sanchez E, Furukawa TA, Taylor J, Martin JL. Systematic review and meta-analysis of cannabis treatment for chronic pain. Pain Med 2009;10:1353-1368.

27. Johnson JR, Burnell-Nugent M, Lossignol D, et al. Multicenter, doubleblind, randomized, placebo-controlled, parallel-group study of the efficacy, safety, and tolerability of THC:CBD extract and THC extract in patients with intractable cancer-related pain. J Pain Symptom Manage 2010;39:167-179.

28. Portenoy RK, Ganae-Motan ED, Allende S, et al. Nabiximols for opioidtreated cancer patients with poorly-controlled chronic pain: a randomized, placebo-controlled, graded-dose trial. J Pain 2012;13:438-449.

29. Hoggart B, Ratcliffe S, Ehler E, et al. A multicentre, open-label, follow-on study to assess the long-term maintenance of effect, tolerance and safety of THC/CBD oromucosal spray in the management of neuropathic pain. J Neurol 2015;262:27-40.

30. Tramer MR, Carroll D, Campbell FA, et al. Cannabinoids for control of chemotherapy induced nausea and vomiting: quantitative systematic review. BMJ 2001;323:16-21.

31. Ben Amar M. Cannabinoids in medicine: a review of their therapeutic potential. J Ethnopharmacol 2006;105:1-25.

32. Barann M, Molderings $G$, Bruss $M$, et al. Direct inhibition by cannabinoids of human 5-HT3A receptors: probable involvement of an allosteric modulatory site. Br J Pharmacol 2002;137:589-596.

33. Meiri E, Jhangiani H, Vredenburgh JJ, et al. Efficacy of dronabinol alone and in combination with ondansetron versus ondansetron alone for delayed chemotherapy-induced nausea and vomiting. Curr Med Res Opin 2007;23:533-543.

34. Duran M, Perez E, Abanades S, et al. Preliminary efficacy and safety of an oromucosal standardized cannabis extract in chemotherapy-induced nausea and vomiting. Br J Clin Pharmacol 2010;70:656-663.

35. Ware MA, Daeninck P, Maida V. A review of nabilone in the treatment of chemotherapy-induced nausea and vomiting. Ther Clin Risk Manage 2008;4:99-107.

36. Davis MP. Oral nabilone capsules in the treatment of chemotherapyinduced nausea and vomiting and pain. Expert Opin Investig Drugs 2008;17:85-95.

37. Navari RM, Nagy CK, Gray SE. The use of olanzapine versus metoclopramide for the treatment of breakthrough chemotherapyinduced nausea and vomiting in patients receiving highly emetogenic chemotherapy. Support Care Cancer 2013;21:1655-1663.

38. Navari RM, Gray SE, Kerr AC. Olanzapine versus aprepitant for the prevention of chemotherapy-induced nausea and vomiting: a randomized phase III trial. J Support Oncol 2011;9:188-195.

39. Todaro B. Cannabinoids in the treatment of chemotherapy-induced nausea and vomiting. J Natl Compr Canc Netw 2012;10:487-492.

40. Roila F, Herrstedt J, Aapro M, et al. Guideline update for MASCC and $\mathrm{ESMO}$ in the prevention of chemotherapy- and radiotherapy-induced nausea and vomiting: results of the Perugia consensus conference. Ann Oncol 2010;21(Suppl 5):v232-243.

41. Smith LA, Azariah F, Lavender VT, et al. Cannabinoids for nausea and vomiting in adults with cancer receiving chemotherapy. Cochrane Database Syst Rev 2015;11:CD009464.

42. Davis MP, Hallerberg G, Palliative Medicine Study Group of the Multinational Association of Supportive Care in Cancer. A systematic review of the treatment of nausea and/or vomiting in cancer unrelated to chemotherapy or radiation. J Pain Symptom Manage 2010;39:756-767.

43. Walsh D, Kirkova J, Davis MP. The efficacy and tolerability of long-term use of dronabinol in cancer-related anorexia: a case series. J Pain Symptom Manage 2005;30:493-495. 
44. Jatoi A, Windschitl HE, Loprinzi CL, et al. Dronabinol versus megestrol acetate versus combination therapy for cancer-associated anorexia: a North Central Cancer Treatment Group study. J Clin Oncol 2002;20:567-573.

45. Cannabis-In-Cachexia-Study-Group, Strasser F, Luftner D, et al. Comparison of orally administered cannabis extract and delta-9. tetrahydrocannabinol in treating patients with cancer-related anorexiacachexia syndrome: a multicenter, phase III, randomized, double-blind, placebo-controlled clinical trial from the Cannabis-In-Cachexia-StudyGroup. J Clin Oncol 2006;24:3394-3400.

46. Navari RM, Brenner MC. Treatment of cancer-related anorexia with olanzapine and megestrol acetate: a randomized trial. Support Care Cancer 2010;18:951-956.

47. Mantovani G, Maccio A, Madeddu C, et al. Randomized phase III clinical trial of five different arms of treatment in 332 patients with cancer cachexia. Oncologist 2010;15:200-211.

48. Nagraj SK, Naresh S, Srinivas K, et al. Interventions for the management of taste disturbances. Cochrane Database Syst Rev 2014;11:CD010470.

49. Brisbois TD, de KockIH, WatanabeSM, etal. Delta-9-tetrahydrocannabinol may palliate altered chemosensory perception in cancer patients: results of a randomized, double-blind, placebo-controlled pilot trial. Ann Oncol 2011;22:2086-2093.

50. Pisanti S, Bifulco M. Endocannabinoid system modulation in cancer biology and therapy. Pharmacol Res 2009;60:107-116.

51. Portella G, Laezza $C$, Laccetti $P$, et al. Inhibitory effects of cannabinoid $\mathrm{CB} 1$ receptor stimulation on tumor growth and metastatic spreading: actions on signals involved in angiogenesis and metastasis. FASEB J 2003;17:1771-1773.

52. Preet A, Qamri Z, Nasser MW, et al. Cannabinoid receptors, CB1 and CB2, as novel targets for inhibition of non-small cell lung cancer growth and metastasis. Cancer Prev Res (Phila) 2011;4:65-75.

53. Hermanson DJ, Marnett LJ. Cannabinoids, endocannabinoids, and cancer. Cancer Metastasis Rev 2011;30:599-612.

54. Marcu JP, Christian RT, Lau D, et al. Cannabidiol enhances the inhibitory effects of delta9-tetrahydrocannabinol on human glioblastoma cell proliferation and survival. Mol Cancer Ther 2010;9:180-189.

55. Guzman M, Duarte MJ, Blazquez C, et al. A pilot clinical study of Delta9. tetrahydrocannabinol in patients with recurrent glioblastoma multiforme. Br J Cancer 2006;95:197-203.
56. Pisanti S, Picardi P, D'Alessandro A, et al. The endocannabinoid signaling system in cancer. Trends Pharmacol Sci 2013;34:273-282.

57. Mukhopadhyay B, Schuebel K, Mukhopadhyay P, et al. Cannabinoid receptor 1 promotes hepatocellular carcinoma initiation and progression through multiple mechanisms. Hepatology 2015;61:1615-1626.

58. Sarnataro D, Pisanti S, Santoro A, et al. The cannabinoid CB1 receptor antagonist rimonabant (SR141716) inhibits human breast cancer cell proliferation through a lipid raft-mediated mechanism. Mol Pharmacol 2006;70:1298-1306.

59. Marshall AD, Lagutina I, Grosveld GC. PAX3-FOXO1 induces cannabinoid receptor 1 to enhance cell invasion and metastasis. Cancer Res 2011;71:7471-7480.

60. Santoro A, Pisanti S, Grimaldi C, et al. Rimonabant inhibits human colon cancer cell growth and reduces the formation of precancerous lesions in the mouse colon. Int J Cancer 2009;125:996-1003.

61. Pisanti S, Picardi P, Prota L, et al. Genetic and pharmacologic inactivation of cannabinoid CB1 receptor inhibits angiogenesis. Blood 2011;117:55415550 .

62. McKallip RJ, Nagarkatti M, Nagarkatti PS. Delta-9-tetrahydrocannabinol enhances breast cancer growth and metastasis by suppression of the antitumor immune response. J Immunol 2005;174:3281-3289.

63. Yuan M, Kiertscher SM, Cheng Q, et al. Delta 9-tetrahydrocannabinol regulates Th1/Th2 cytokine balance in activated human $\mathrm{T}$ cells. J Neuroimmunol 2002;133:124-131.

64. Van den Hove LE, Van Gool SW, Van Poppel H, et al. Phenotype, cytokine production and cytolytic capacity of fresh (uncultured) tumourinfiltrating $\mathrm{T}$ lymphocytes in human renal cell carcinoma. Clin Exp Immunol 1997;109:501-509.

65. Pardoll DM. The blockade of immune checkpoints in cancer immunotherapy. Nat Rev Cancer 2012;12:252-264.

66. Taube JM, Klein A, Brahmer JR, et al. Association of PD-1, PD-1 ligands, and other features of the tumor immune microenvironment with response to anti-PD-1 therapy. Clin Cancer Res 2014;20:5064-5074.

67. Ruchlemer R, Amit-Kohn M, Raveh D, Hanus L. Inhaled medicinal cannabis and the immunocompromised patient. Support Care Cancer 2015;23:819-822. 